\title{
Enhanced Impact Energy Absorption Characteristics of SandwichComposites through Tufting
}

\author{
Anamaría Henao ${ }^{1}$, Roberto Guzmán de Villoria ${ }^{2}$, Jesús Cuartero ${ }^{1}$, Marco \\ Carrera $^{1}$, Juan Picón $^{3}$, Antonio Miravete ${ }^{4}$ \\ ${ }^{1}$ Departamento de Ingeniería Mecánica \\ Universidad de Zaragoza \\ Calle Maria de Luna S/N, Zaragoza, Spain \\ e-mail: henaoanamia@hotmail.com \\ ${ }^{2}$ IMDEA Materials Institute \\ $\mathrm{C} /$ Profesor Aranguren, $\mathrm{s} / \mathrm{n}$. \\ 28040, Madrid, Spain \\ ${ }^{3}$ Metro de Madrid S.A. \\ C/ Cavanilles 58 \\ 28007 Madrid, Spain \\ ${ }^{4}$ Instituto de Ciencia de Materiales de Aragón \\ Sede Campus Río Ebro \\ C/ María de Luna, 3 \\ 50018 Zaragoza, Spain
}

\section{Keywords}

Sandwich structures, Energy absorption, Tufting

\begin{abstract}
Sandwich structures, which are made of a core material bonded to two face sheets on both sides, are highly demandedwhere a high flexural stiffness per weight ratio is needed. The main limiting factor of these materials is the core and the face sheets interface, which tends to delaminate. Tufting is one of the most promising technologies to reinforce this interface along the z-direction. Here, the energy absorption of tufted sandwich structure under impact loads is evaluated. Six different types of tufted specimens were tested, including both carbon and glass fiber faces with three different tufting densities. We demonstrate here that the impact behavior of sandwich structure is effectively improved by the tufting process, and that the higher the density of the tufted sandwiches, the higher the energy absorbed
\end{abstract}

\section{Introduction}

For the last few years, an increasing interest in the application of composite components in automotive and rail applications has emerged. In both fields, impact energy absorption along stiffness and strength are usually required in 
order to be competitive with metallic materials. Among the composite materials, sandwich structures are, in many ways, good candidate configurations to compete against metals. However, their use has been limited mainly because of their poor skin/core interface properties. In order to overcome this problem, innovative research lines have arised and developed the insertion of fibers through the sandwich structure, using technologies such as tufting or stitching. This study focuses on the impact behavior of tufted sandwich structures, which have been proven to be efficient in terms of stiffness and strength [1].

The advantages of using fibers through the sandwich have been highlighted by others [2-5]. Among these technologies, tufting, stitching and Z-pinning are the most promising for industry applications.

The tufting method is based on conventional stitching process which forms the seams by using dual-threading system forming loops and knots; this kind of union causes an important deterioration over the mechanical performance of the $3 \mathrm{D}$ structure. The innovative aspect of the tufting process is the use of a single needle that brings into the structure a thread without tension. This results in an improvement of in-plane properties over the stitching process. A consolidated structure is obtained after the resin impregnation by using the Vacuum Assisted Resin Transfer Molding (VARTM) process; this structure has higher properties that one without reinforcement through the thickness [1].

In the tufting process a thread is brought into the preform by one needle with an angle between $90^{\circ}$ to $45^{\circ}$. The thread is retained into the preform by friction between the fabrics or foam with the thread, forming a loop. The difference between the complex stitching (left) and the simple tufting scheme (rigth) is showed in Fig. 1.

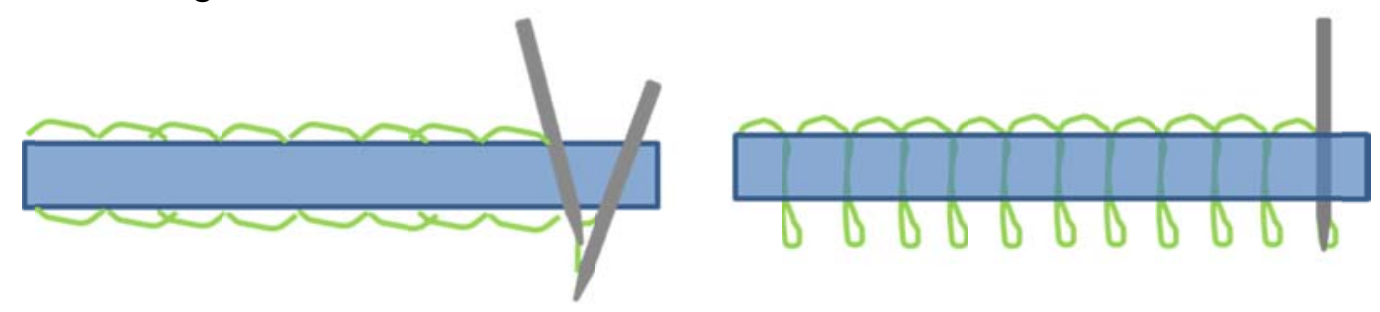

Figure1. Schemes of stitching (left) and tufting (left) applied to sandwich panels.

In order to provide enough robustness during the tufting process a needle of $2 \mathrm{~mm}$ is used to develop repeated applications. The dry preform used in this study is composed by two faces made out of glass or carbon fabrics and the foam core. After that, all the components are assembled into a dry sandwich structure by tufting with the parameters being previously set. This process compared with stitching machine doesn't produce 'locked' and the thread only remains in position in consequence of frictional forces between the thread and 
the preform. Consequently, tufting process produces a virtually tension-free structure with less waviness in the fabric and fewer problems during resin injection process.

Another application of tufting is used to join semi-finished parts together, in order to make the laid up material easier to handle. This one-side access technique requires less tooling compared to standard stitching, providing also the possibility to apply tufting directly on the mold, to process complex and 3D shaped pre-forms, unlimited thickness by subsequent lay ups and to choose needle penetration angle.

The robot used in the development of the present work is showed in figure 2, the used of a robot-assisted tufting gives the opportunity for localized reinforcement, the possibility of rapid processing of complex three dimensional shapes, high reproducibility, in less time, with virtually no manual handling of the fabric before resin injection.

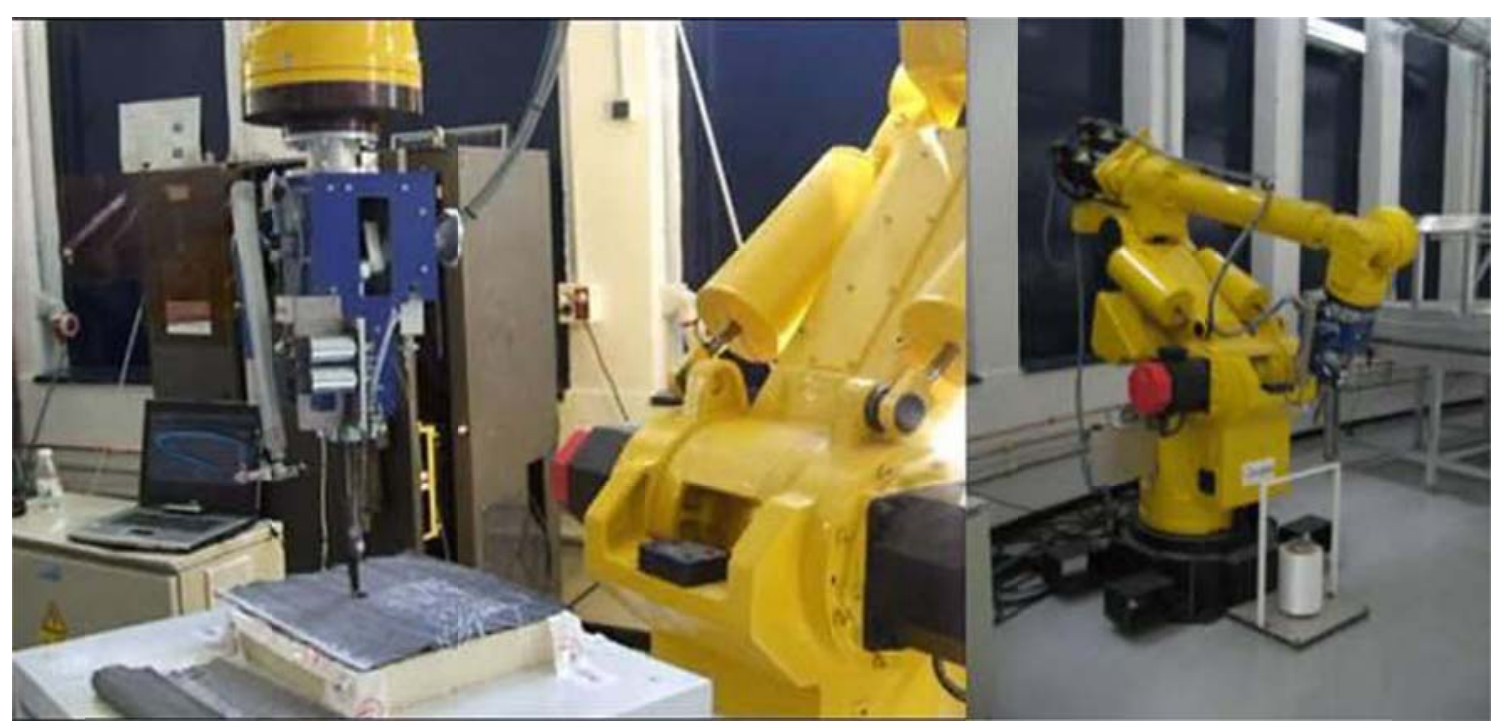

Figure 2.Fanuc 6-axis robot and tufting head.

Otherwise, the stitching process [6-10], differently to the tufting, may generate rich resin areas and potential fiber breakage due to the nodes produced during the stitching processing. On the other hand, the stitching technology requires open areas between the inferior and the superior parts of the stitching machine. To overcome this problem, an accurate synchronization system is needed [11], which makes the stitching a more complex technology than tufting. Essentially tufting is equivalent to z-pinning, but the tufting technology presents the advantage of applying the reinforcing dry fabrics prior to resin injection using Vacuum Assisted Resin Transfer Moulding(VARTM) processes.

\section{Experiment}




\section{Materials}

Two material systems have been used for the sandwich faces: carbon/epoxy and E-glass/epoxy. In terms of core materials, $35 \mathrm{~kg} / \mathrm{m} 3$ polyurethane (PUR) foams have been utilized. Both tufted and non-tufted sandwich panels have been studied under impact loads.

The E-glass fabric used for the faces was $800 \mathrm{~g} / \mathrm{m} 2$ [0/90] woven fabrics supplied by OCV. The carbon fiber was also a woven fabric with a density of $800 \mathrm{~g} / \mathrm{m} 2$ and a layup of [0/90] as well made by OCV from Toray carbon fiber T700S. An E-glass threadEC9 68X3 S260 with 2400 tex supplied by Tissafil was used to make tufting in sandwich structures. This fiber has been used in these types of applications for some time. An Aralidte LY1564 SP epoxy resin with hardener XB3487 was used as matrix, both supplied by Hunstman.

\section{Tufting process}

The tufting process has been applied by means of a tufting head (KSL KL 150), controlled by a Fanuc 6-axis robot, figure 2. Variables such as stitch length, insertion angle and the length of the loop were defined and controlled during the process. During this studies a $45^{\circ}$ angle was applied and three different stitch length 20,30 and $40 \mathrm{~mm}$.

A needle of $2 \mathrm{~mm}$ diameter was used to provide enough robustness to develop repeated applications. The dry perform is formed by two faces made of fabrics and the core made of a polyurethane foam. A dry sandwich structure is then assembled by tufting. Subsequently, the impregnation of the sandwich structure was carried out by means of the Vacuum Assisted Resin Transfer Moulding (VARTM) process.

\section{Impact test}

The tufting influence over sandwich structures was measured by performing impact test, followed by damage evaluation of the different configuration specimens.

A Ceast Fractovis Drop Weight Impact Testing Machine equipped with a data acquisition system was used to conduct the impact tests. The steel hemispherical impactor with a diameter of $20 \mathrm{~mm}$ was adopted for all tests, the $120 \mathrm{~mm} \times 120 \mathrm{~mm}$ samples were placed between the clamps. The specimen support fixture at the bottom of the drop tower facilitates a square clamped condition with a clear span of $81 \mathrm{~mm} \times 81 \mathrm{~mm}$. An impact energy level of $40 \mathrm{~J}$ was used. In addition, the load-time histories for all the samples were recorded.

Tables 1 and 2 show the main characteristic of the different specimen groups. 
Table 1.Sandwich structures with carbon fiber faces characteristics

\begin{tabular}{|c|c|c|c|c|}
\hline Group & $\begin{array}{c}\text { Distantance } \\
\text { between } \\
\text { tufted lines } \\
(\mathrm{mm})\end{array}$ & $\begin{array}{c}\text { Averagethickness } \\
(\mathrm{mm})\end{array}$ & $\begin{array}{c}\text { Averageweigth } \\
(\mathrm{g})\end{array}$ & $\begin{array}{c}\text { Weight per } \\
\text { average } \\
\text { area unit } \\
\left(\mathrm{kg} / \mathrm{m}^{\wedge} 2\right)\end{array}$ \\
\hline IC1 & - & 33.61 & 98 & 6.82 \\
\hline IC2 & 40 & 34.22 & 111 & 7.72 \\
\hline IC3 & 30 & 34.26 & 114 & 7.89 \\
\hline IC4 & 20 & 34.29 & 124 & 8.64 \\
\hline
\end{tabular}

*Specimen length is $120 \mathrm{~mm}$

Table 2.Sandwich structures with glass fiber faces characteristics

\begin{tabular}{|c|c|c|c|c|}
\hline Group & $\begin{array}{c}\text { Distantance } \\
\text { between } \\
\text { tufted lines } \\
(\mathrm{mm})\end{array}$ & $\begin{array}{c}\text { Averagethickness } \\
(\mathrm{mm})\end{array}$ & $\begin{array}{c}\text { Averageweigth } \\
(\mathrm{g})\end{array}$ & $\begin{array}{c}\text { Weight per } \\
\text { average area } \\
\text { unit } \\
\left(\mathrm{kg} / \mathrm{m}^{\wedge} 2\right)\end{array}$ \\
\hline IG1 & - & 32.15 & 95 & 6.67 \\
\hline IG2 & 40 & 33.35 & 113 & 7.91 \\
\hline IG3 & 30 & 33.51 & 116 & 8.08 \\
\hline IG4 & 20 & 33.81 & 127 & 8.82 \\
\hline \multicolumn{5}{|r|}{ *Specimenlengthis $120 \mathrm{~mm}$} \\
\end{tabular}

\section{Scanning electron microscopy (SEM)}

Micrographs were taken during the test using the equipment Quanta 200 SEM, which is a versatile high performance, low-vacuum scanning electron microscope with a tungsten electron source. It is delivered with a $50 \mathrm{~mm}$ (2 inch) motorized stage (x/y travel), with a motorized z-range of $25 \mathrm{~mm}$, accelerating voltage from $0.2 \mathrm{a} 30 \mathrm{Kv}$, resolution of $3.5 \mathrm{~nm}$.

\section{X-ray evaluation}

Every sandwich typology was studied by X-ray using Metris X-TekHMX ST 225 CT scanner equipment. A X-ray opaque dye solution was applied over the impacted sandwich surface in order to visualize the damaged area.

\section{Results and discussion}

\section{Sandwiches with carbon fiber faces impact testing results}


Drop weight impact tests were performed on sandwich structures with carbon fiber faces in order to study the effect of fiber insertion through the thickness on the impact response. At least 5 specimens of each tufting condition were tested.

Impact forces and deflections were recorded as a function of time during each impact event, the height of the impactor was also recorded in order to calculate the impact energy as a function of time. All the data were recorded every 6 milliseconds.

Tabulated values for the average impact force, absorbed energy and deflection during the impact event for all typologies are presented in Table 3. These results show that the absorbed energy increases with the increasing of the tufting density; for the highest tufting density (IC4) the energy increases about $18 \%$ against sandwich without tufting; for typologies IC 3 , with a tufting density of $30 \mathrm{~mm}$, the increasing was $14 \%$ and for the lowest density (IC2) the energy increases $11 \%$ more than the sandwich without tufting.

Table 3also shows that the addition of tufting in the sandwich structure tested increased the contact force. However the increase of the tufting density reduced the contact force increase. Therefore, in the case of lowest density (IC2) the increased contact force was estimated to be of 22\%; in typology IC3 with tufting density of $30 \mathrm{~mm}$ of $20 \%$ and finally for the highest density IC4 the contact force was increased by $18 \%$.

We also observed a reduction of deflection with the increase of tufting density, due to the higher rigidity obtained by the fiber insertion (Table 3). In the case of the highest density, the reduction in deflection was of approximately $17 \%$. In typologies IC3 and IC2 this reduction was of $11 \%$ and $13 \%$ respectively.

Table 3. Average results of sandwich structures with carbon fiber faces

\begin{tabular}{|c|c|c|c|}
\hline Group & $\begin{array}{c}\text { Average } \\
\text { absorbed } \\
\text { energy (J) }\end{array}$ & $\begin{array}{c}\text { Contactforce } \\
(\mathrm{N})\end{array}$ & $\begin{array}{c}\text { Deflection } \\
(\mathrm{mm})\end{array}$ \\
\hline IC1 & 25.16 & 5018 & 12.85 \\
\hline IC2 & 26.56 & 6131 & 11.12 \\
\hline IC3 & 28.74 & 6036 & 11.41 \\
\hline IC4 & 29.85 & 5927 & 10.56 \\
\hline
\end{tabular}

We then measured displays the energy against time curves for the four different types of sandwich structure with carbon fiber faces (Figure 4). We observed that each curve increased with time during the loading, reached a maximum value and then decreased during unloading, and finally remained horizontal at a constant value. This constant value gives the total energy absorbed 
permanently by the specimen at the end of an impact event. The maximum value of each curve represents the impact energy. The difference between the absorbed energy and impact energy is termed excessive energy. The excessive energy is retained in the impactor and used to rebound the impactor from the non-perforated specimens. (40)

The comparison between all specimens in figure 3 shows that an increasing of tufting density rises the absorbed energy that involves a larger damage in the structure. This behavior is also shown in the SEM micrographs and X-ray images show in figures 8 and 9 respectively.

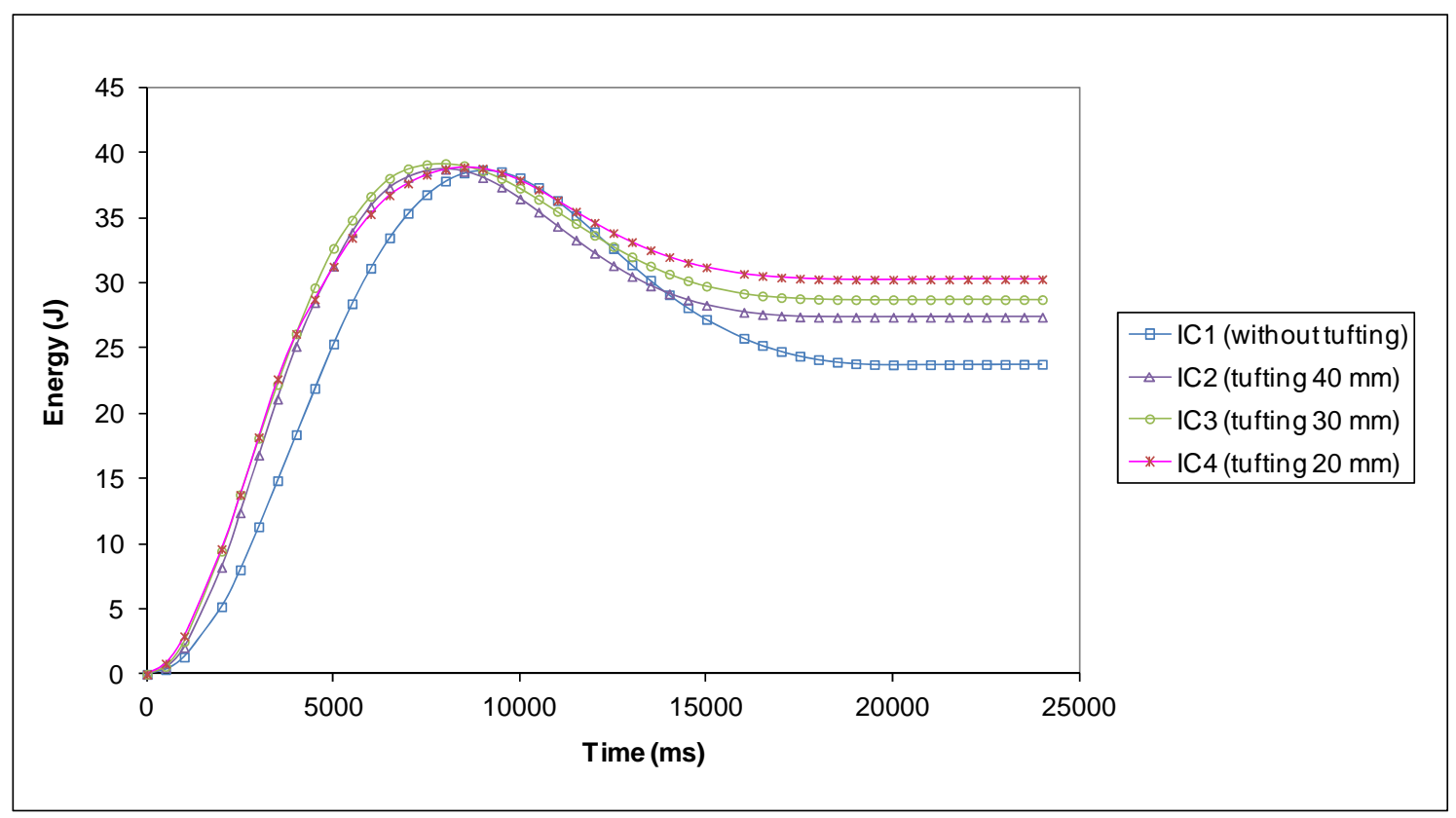

Figure 3.Energy vs time for carbon fiber face sandwich

Figure 4 shows the comparison between the contact force against time for tufted and no-tufted sandwich structures. In the case of tufted sandwich, the contact force is on average $18 \%$ bigger than the non-tufted sandwich. However, there is no significant improvement with the increasing of tufting density. When the absorbed energy is low, such as in specimens without tufting (IC1), the contact force-time curves are of parabolic shape. However, for tufted structures such as IC2, IC3 and IC4, this curve has a peak, that it is more drastic in the case of higher tufting densities, such as IC4, that involves a serious damage of the specimen. 


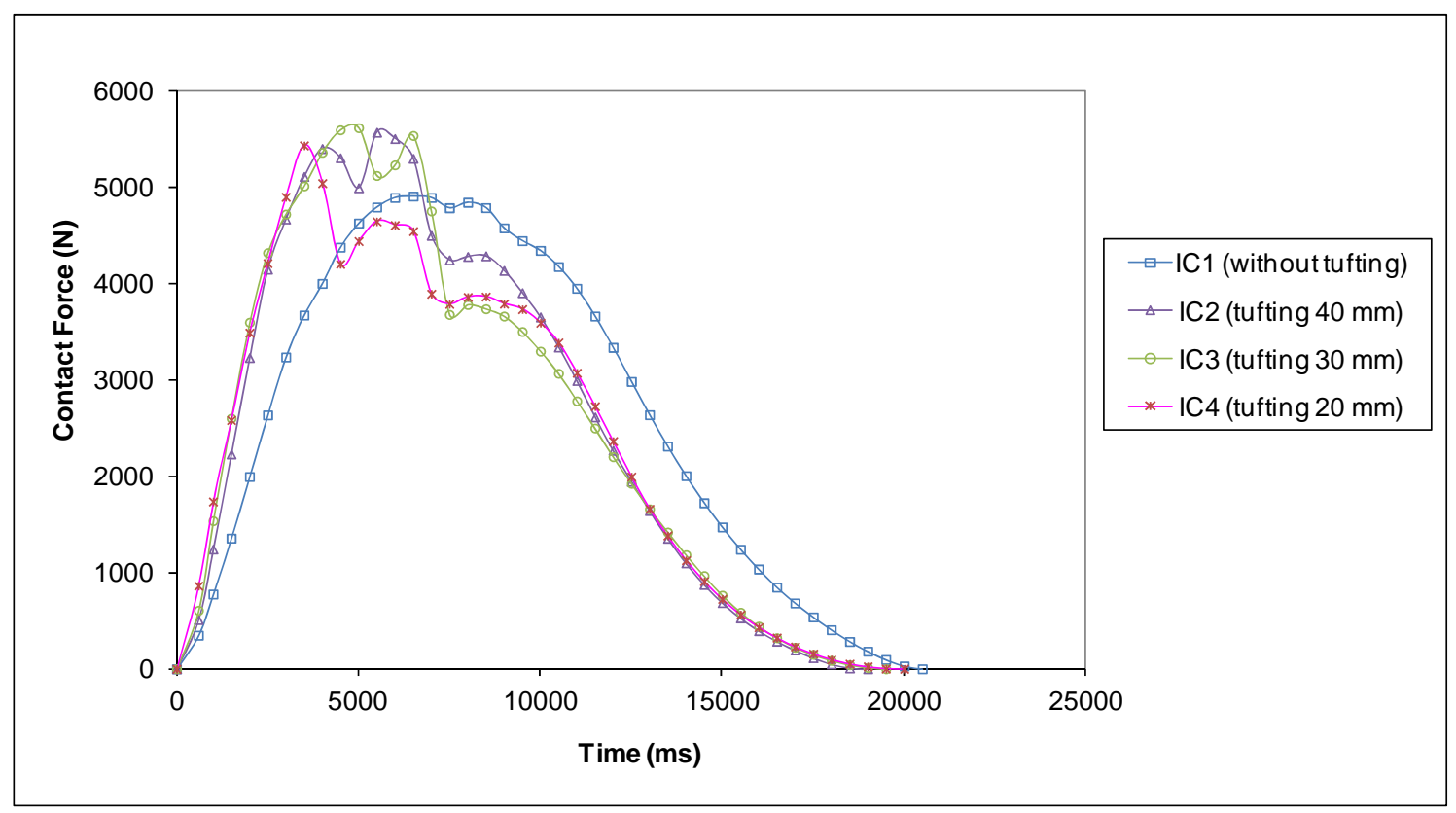

Figure 4. Contact force vs time for carbon fiber face sandwich

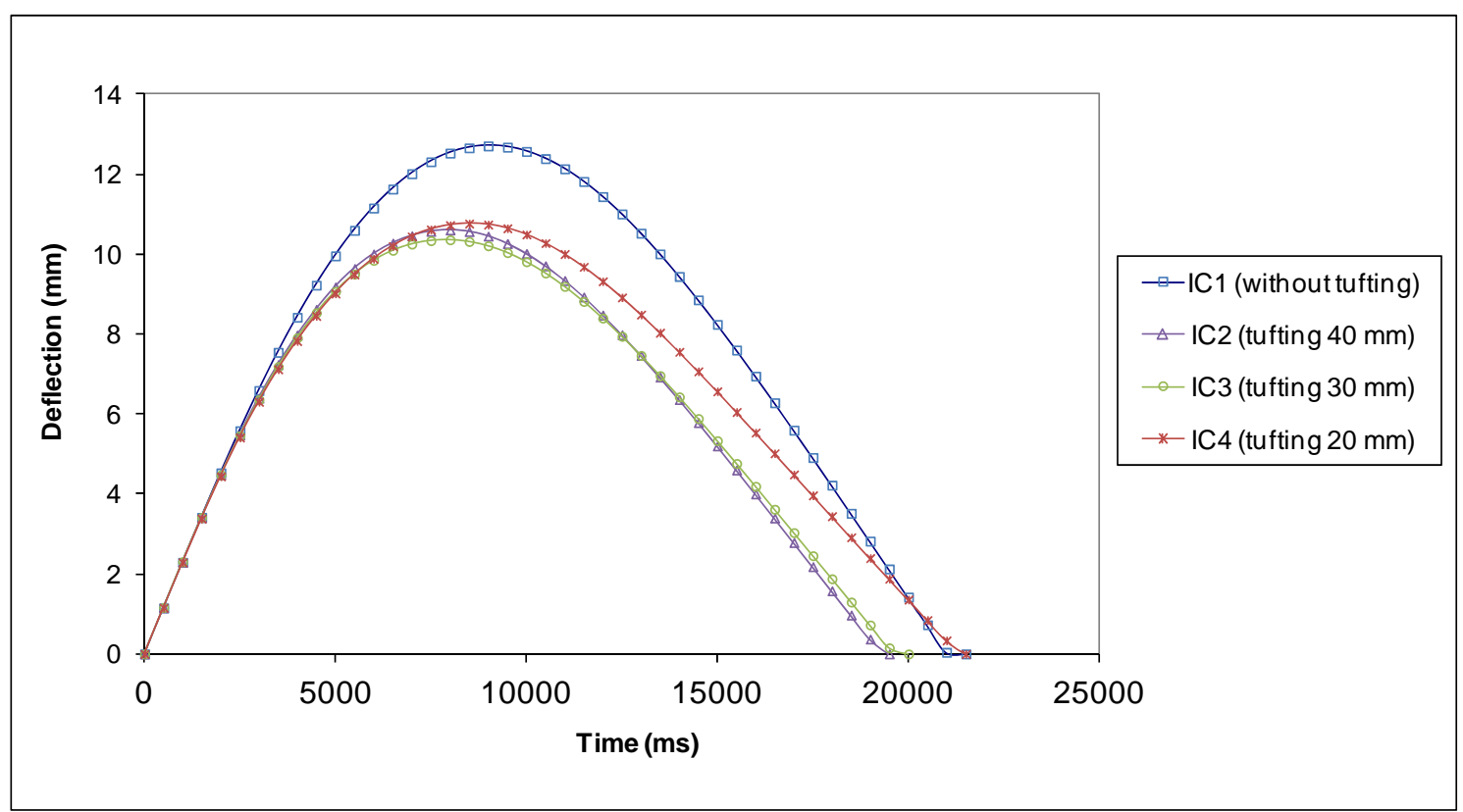

Figure 5. Deflection vs time for carbon fiber face sandwich

From the comparison of contact force-time curves (figure 4) and deflection-time curves (figure 5), we observed that it takes more to reach maximum deflection compared to contact force in the case of tufted specimens. That is, it takes longer for the impactor to return to its initial position, as the absorbed energy increases. This suggests that there is a larger amount of damaged fibers due to increased of absorbed energies.

Figure 6shows the signature of a sandwich structures response to impact. The contact force can be defined as the compressive load that the specimens apply over the impactor. In this study all the obtained curves are closed, due to any of 
the specimens was perforated, and therefore, the entire descending section consists of rebounding, because both the load and deflection decrease.

In the case of sandwich without tufting (IC1), the contact force-deflection curve is smooth, and it does not reach a plateau around the peak force because in this case the impact load does not result in serious damage of the specimens. However in tufted structures (IC2, IC3 and IC4), this curve presents a plateau around the peak force which implies that a larger number of fibers have been damaged due to the bending, structure debonding and loss of structure rigidity. This damage is represented by a steep drop in the curve, as it is observed in specimens with medium and high tufting density (IC3 and IC4). That steep drop suggests the loss of contact between the impactor and the specimen for a moment. As the absorbed energy continues to increase, such as in of structure with the highest tufting density, IC4, the contact force-deflection curves become open, instead of closed one and represents the initiation of perforation.

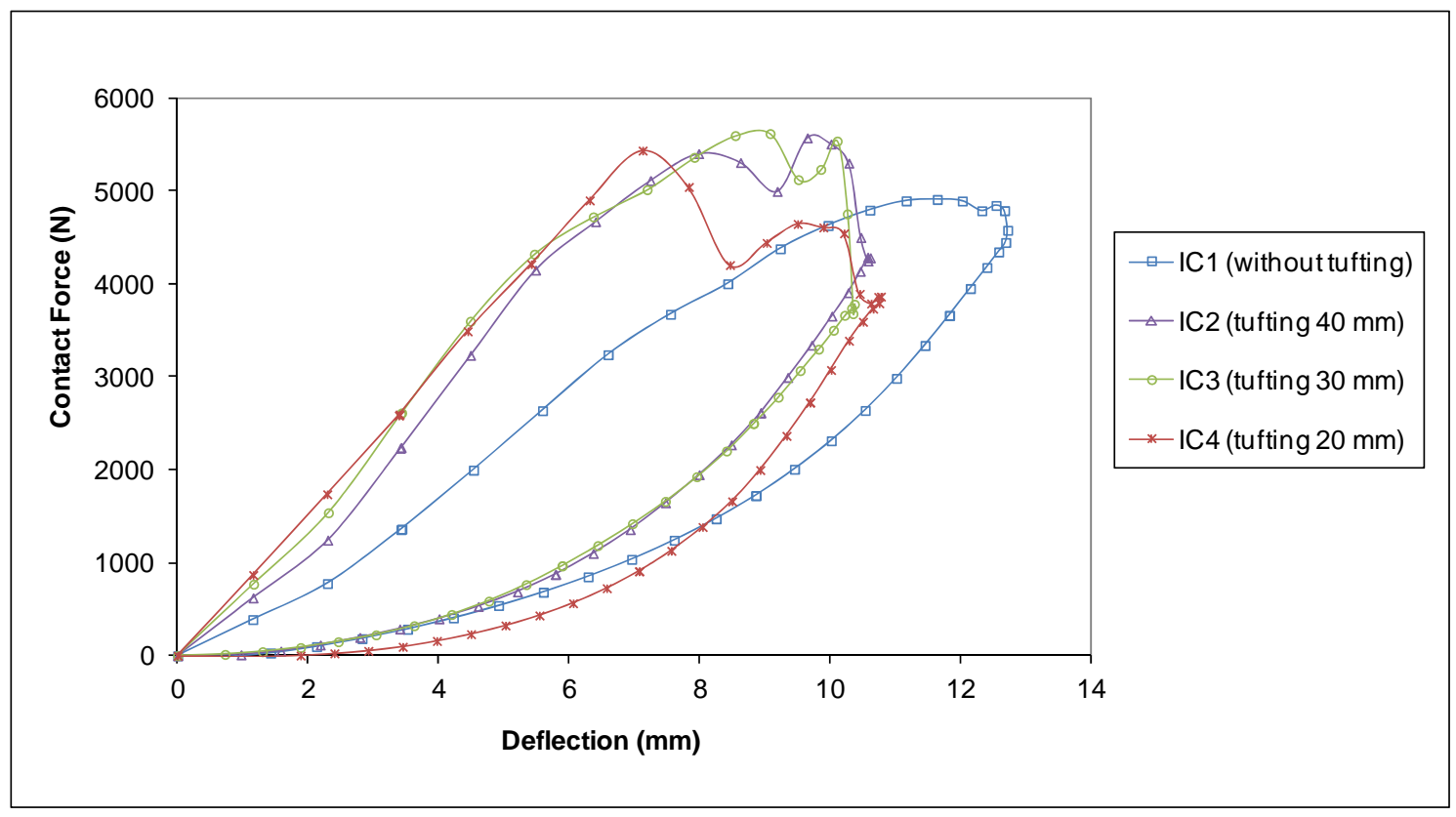

Figure 6. Contact force vs deflection for carbon fiber faces sandwich

Following impacting, the impacted areas within the specimens were nondestructively examined using SEM analysis and X-ray inspection.

Figure 7 shows 23X SEM micrographics of impacted surface of sandwich without and with tufting. As expected, the tufted structure showed more damage than the sample with no tufting (IC1) due to the fact that it absorbed more impact energy. 


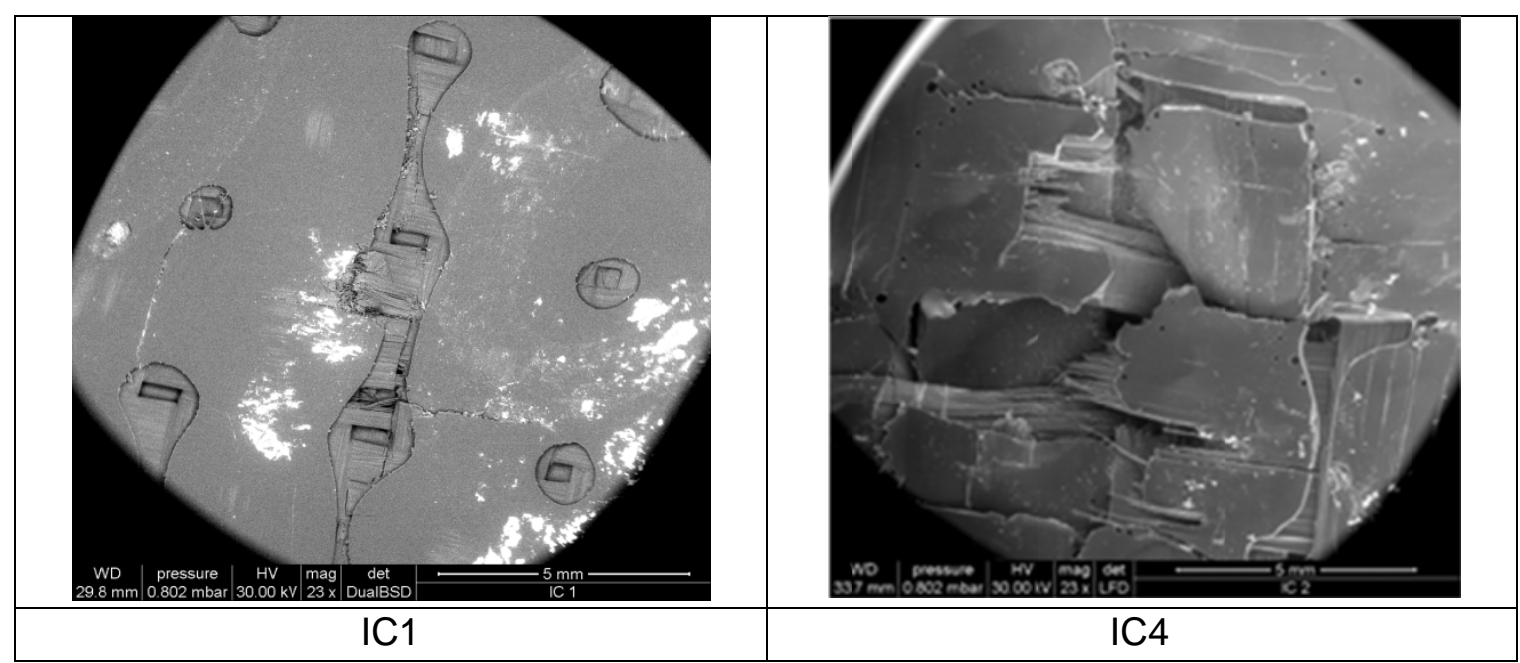

Figure 7. SEM micrographics of each kind of typology of carbon fiber face sandwich without tufting and with tufting

X- ray photographs of the specimens with and without tufting subjected to an impact are presented in Figures 8 (frontal) and 9 (lateral). These photographs demonstrate the behavior described in the graphs and SEM analysis.

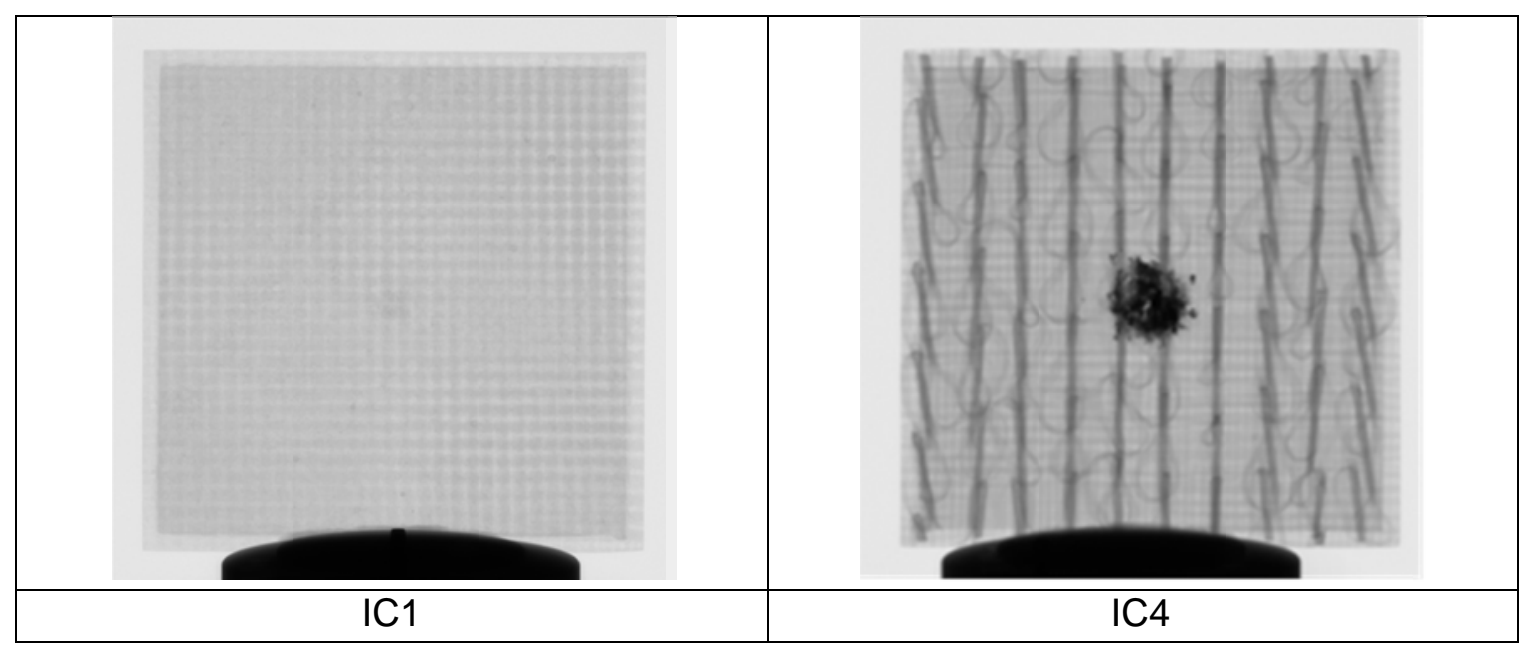

Figure 8. X- ray frontal photographs of the different typologiesof carbon fiber faces sandwich without (left) and with (right) tufting 


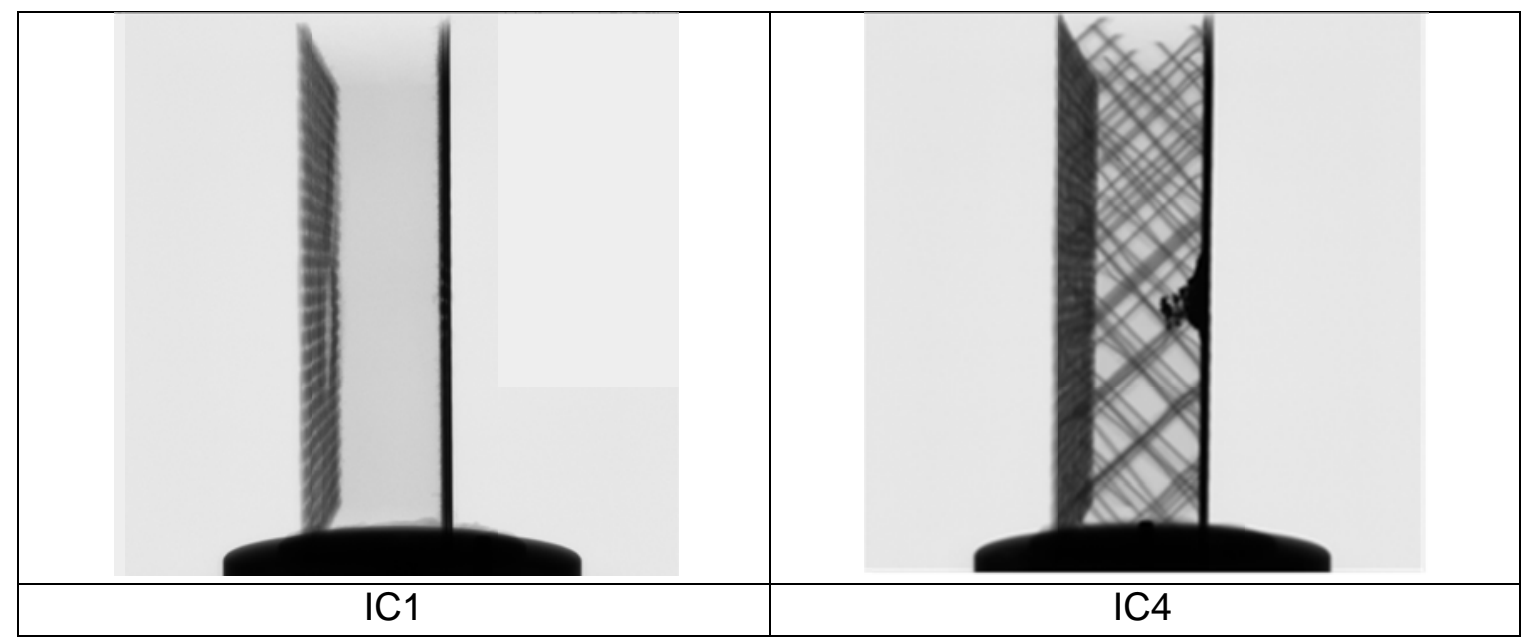

Figure9. X- ray lateral photographs of the different typologies of carbon fiber face sandwich without (left) and with (right) tufting

\section{Sandwiches with glass fiber faces impact testing results}

Table 4 presents the average results obtained during the impact test over sandwich structures with glass fiber faces, with or without tufting. These results show an increase of absorbed energy as the tufting density increases. For specimens with highest density, IG4, the increase is of $27 \%$, in typology IG3, medium density, of $26 \%$ and for lowest density the absorbed energy increased of $20 \%$ in comparison with sandwich without tufting.

In terms of the contact force, there is a generalized increase in all tufted specimens of about $29 \%$ with respect to the non-tufted coupons, however there are is not any significant rise when increasing the tufting density.

The deflection under impact load is also shown in table 4, in this aspect the deflection decrease about $19 \%$ with the insertion of fiber through the thickness.

Table 4.Average results of sandwich structures with glass fiber faces

\begin{tabular}{|c|c|c|c|}
\hline Group & $\begin{array}{c}\text { Average } \\
\text { absorbed } \\
\text { energy (J) }\end{array}$ & $\begin{array}{c}\text { Contact } \\
\text { force } \\
(\mathrm{N})\end{array}$ & $\begin{array}{c}\text { Deflection } \\
(\mathrm{mm})\end{array}$ \\
\hline IG1 & 27.64 & 3954 & 16.31 \\
\hline IG2 & 33.44 & 5075 & 13.39 \\
\hline IG3 & 34.93 & 5126 & 13.76 \\
\hline IG4 & 35.15 & 5030 & 13.20 \\
\hline
\end{tabular}

The variation of the absorbed energy by different tufting density, with real time is presented in figure10. The amount of absorbed energy at the end of impact events increased with the insertion of fiber through the thickness, since a more rigid structure resulted in a more severe damage. High and medium tufting 
densities presented similar behavior, however, a reduction of the density produced lower damage and lower absorbed energy levels.

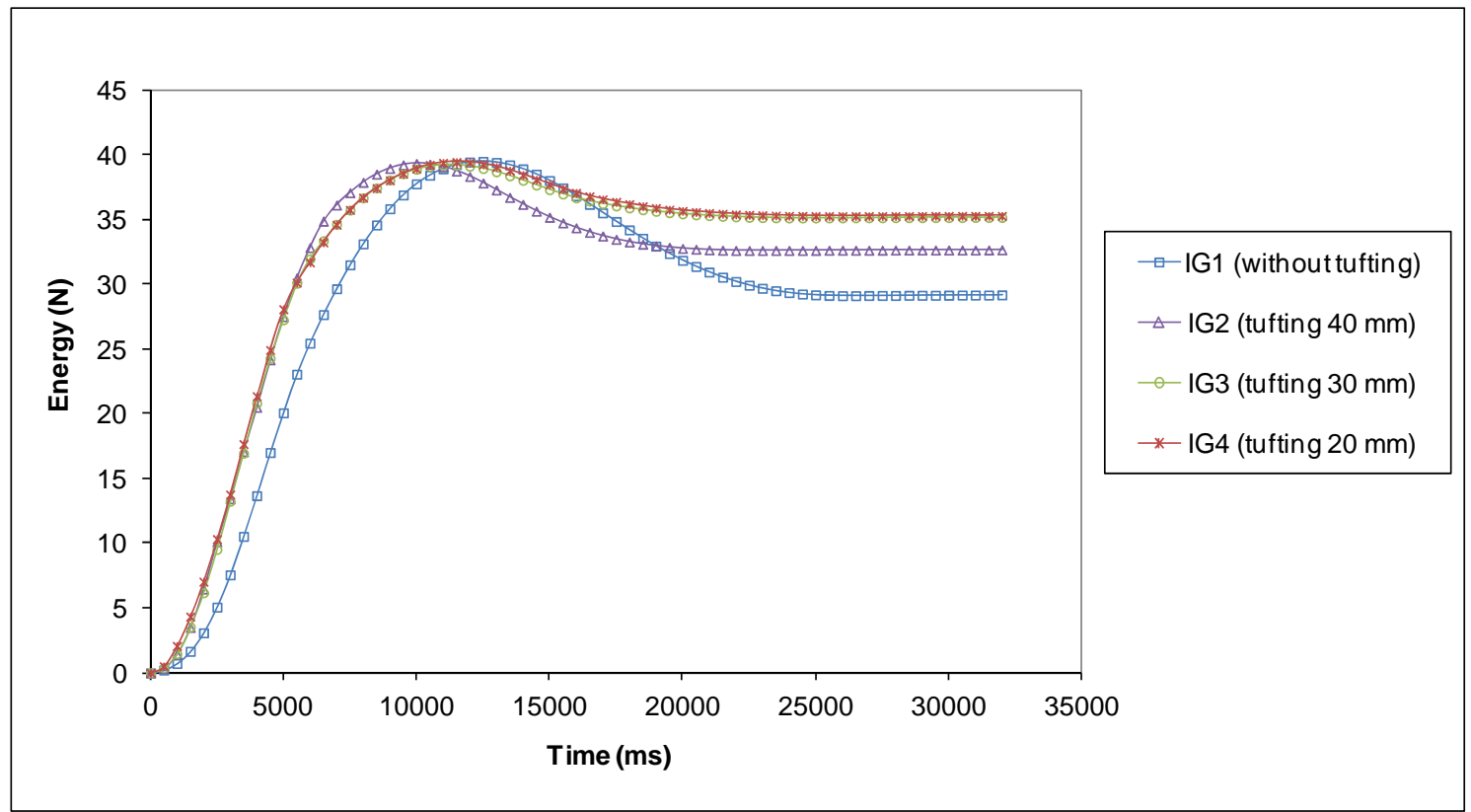

Figure 10. Energy vs time for carbon fiber face sandwich

Figure11 displays four contact force-time $(F-t)$ curves one for each sandwich structure typology. These curves present a similar behavior than the carbon fiber ones. For the sandwich structure without tufting the shape is parabolic whereas for sandwiches with tufting, the curves present a peak that represents a severe damage in the specimen.

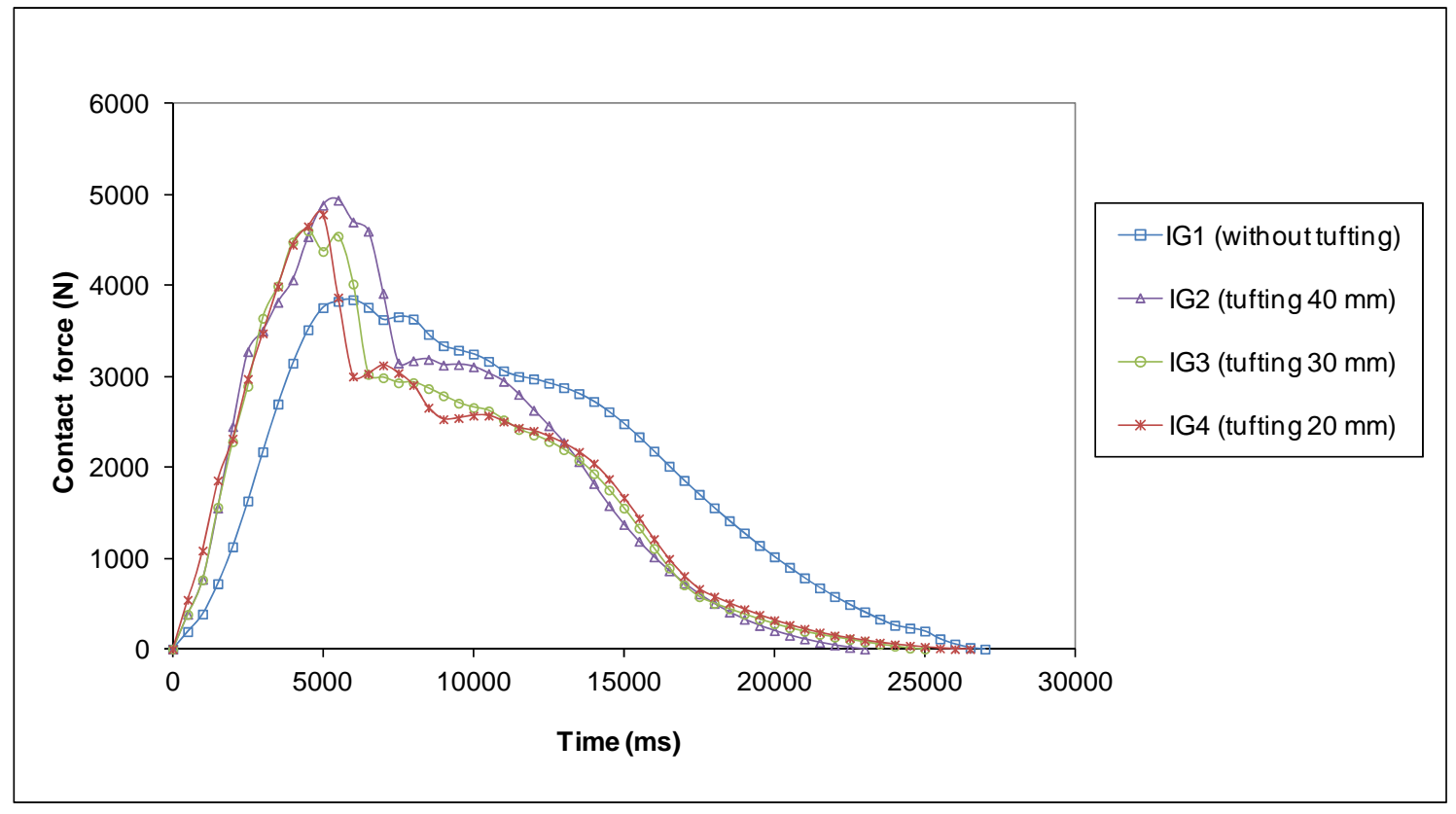

Figure 11. Contact force vs time for glass fiber face sandwich 
Figure 12 shows the deflection-time $(\mathrm{d}-\mathrm{t})$ curves of every different kind of sandwich structure specimens. The comparison of contact force-time curves (Figure 13) and deflection-time curves (Figure14), it is also observed as in the carbon fiber structures that it takes more to reach maximum deflection compared to contact force in the case of tufted specimens.

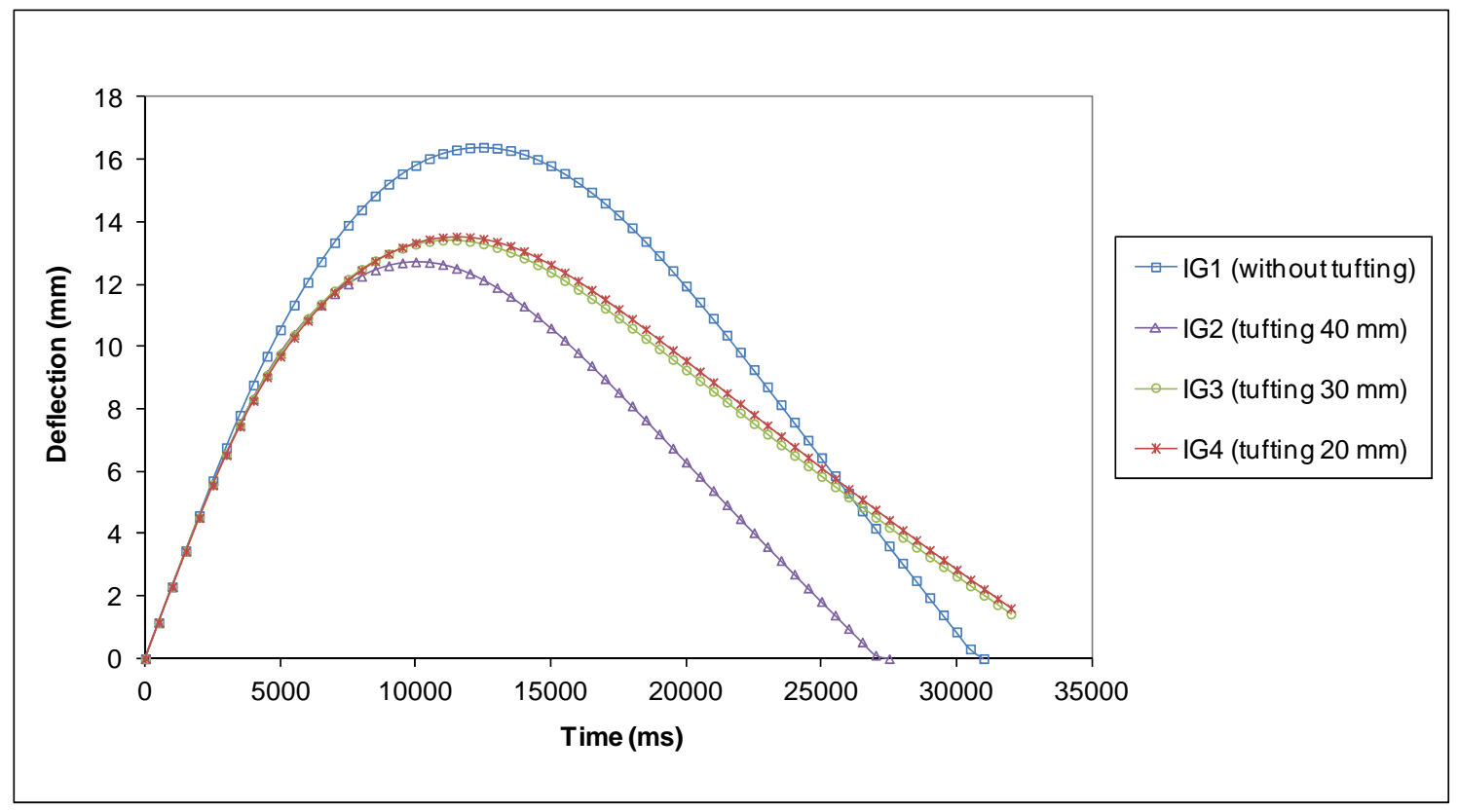

Figure 12. Deflection vs time for glass fiber face sandwich

The biggest difference between carbon fiber and glass fiber specimens is presented in the contact force-deflection $(F-d)$ curves under impact (Figures6 and 13 respectively)because all the glass fiber sandwich structure has plateau instead of peak, that is an indication of rigidity loss, fiber fracture and debonding. The glass fiber structures also present more opened curves than the ones presented for carbon fibers. 


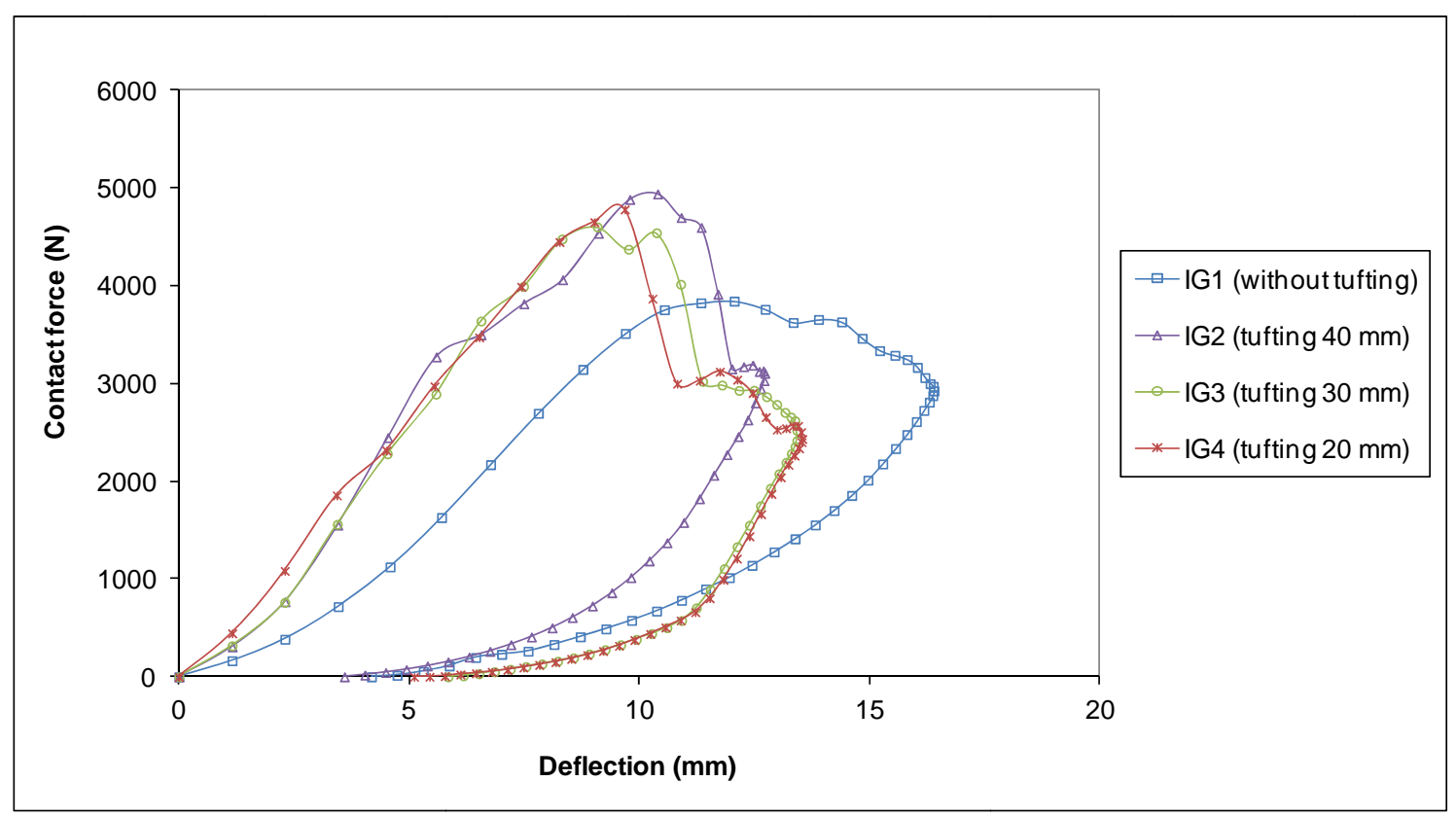

Figure 13. Contact force vs deflection for glass fiber faces sandwich

Figure 14 displays micrographics took during SEM analysis for glass fiber facesspecimens without and with tufting.

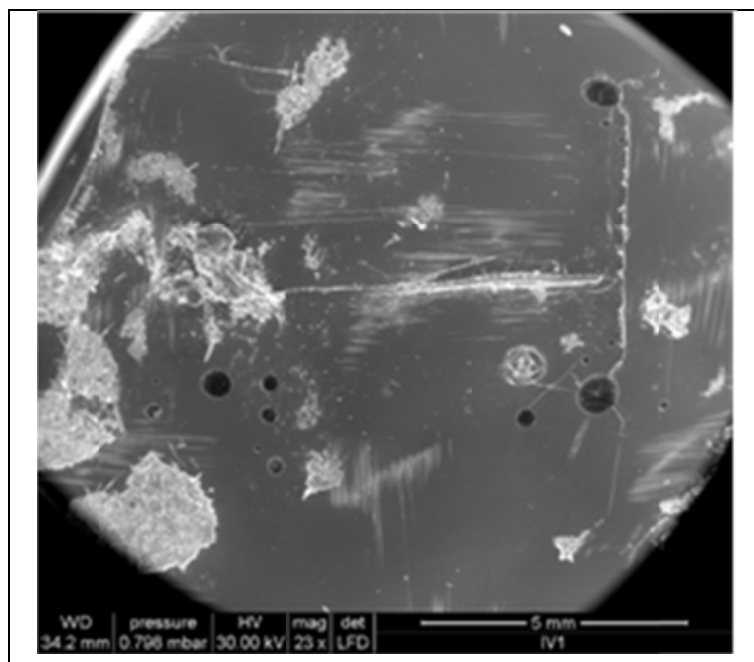

IV1

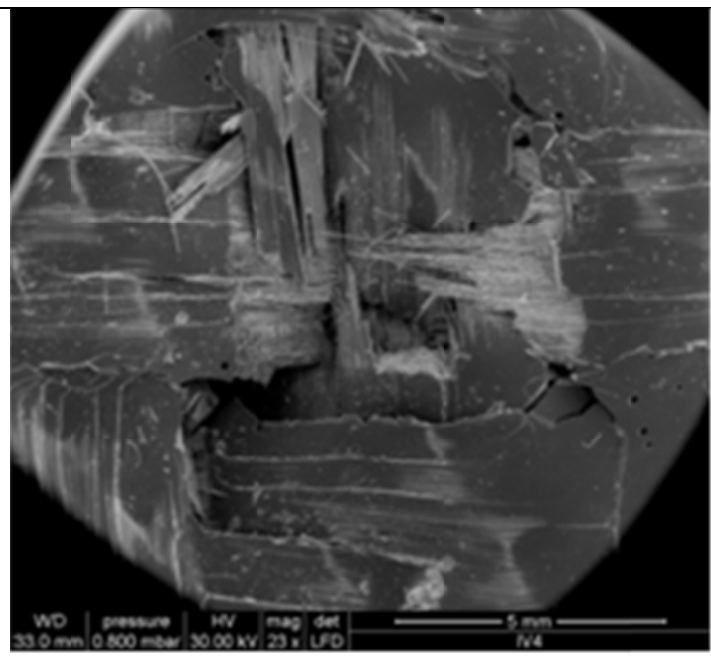

IV2

Figure 14. SEM micrographs of each kind of typology of glass fiber face sandwich without (left) and with (right) tufting

Comparisons between the X-ray photographs are presented in Figure 15 and 16 for all sandwich structure with glass fiber faces with or without tufting. 


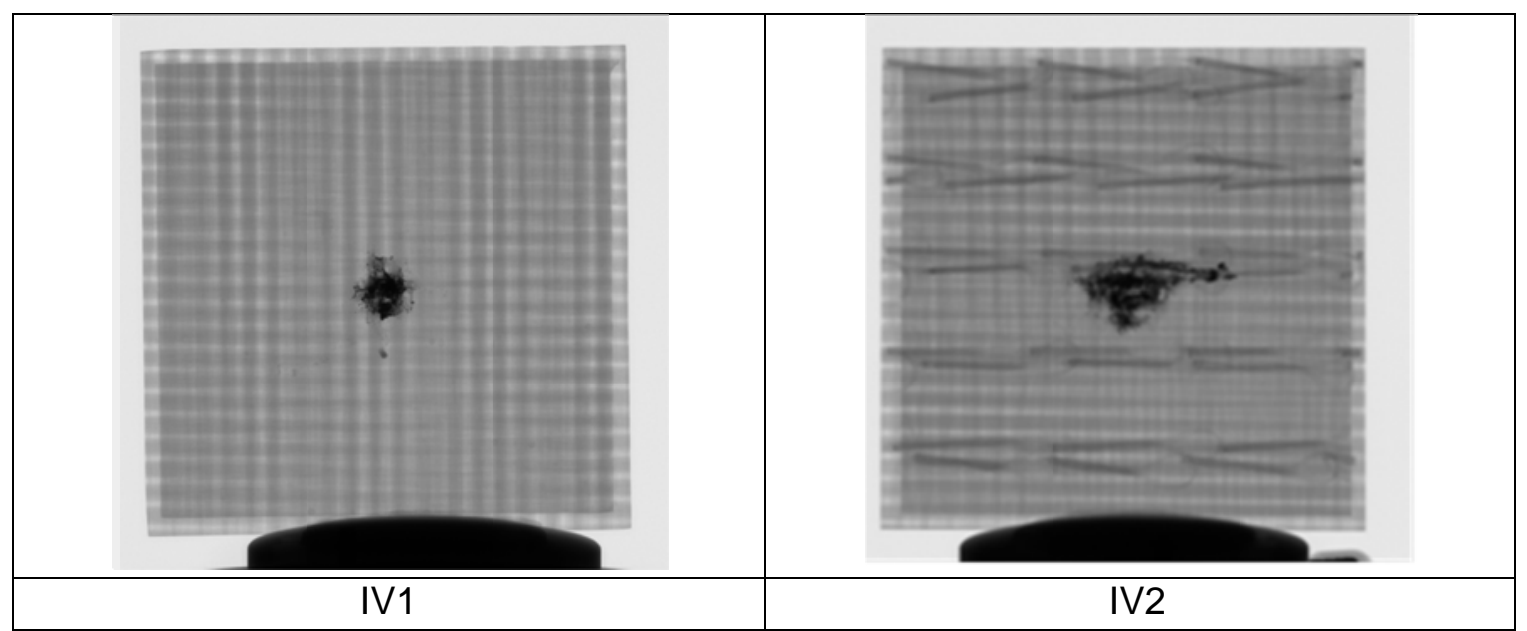

Figure 15. X- ray frontal photographs of the different typologies of gllass fiber face sandwich without (left)and with (right) tufting

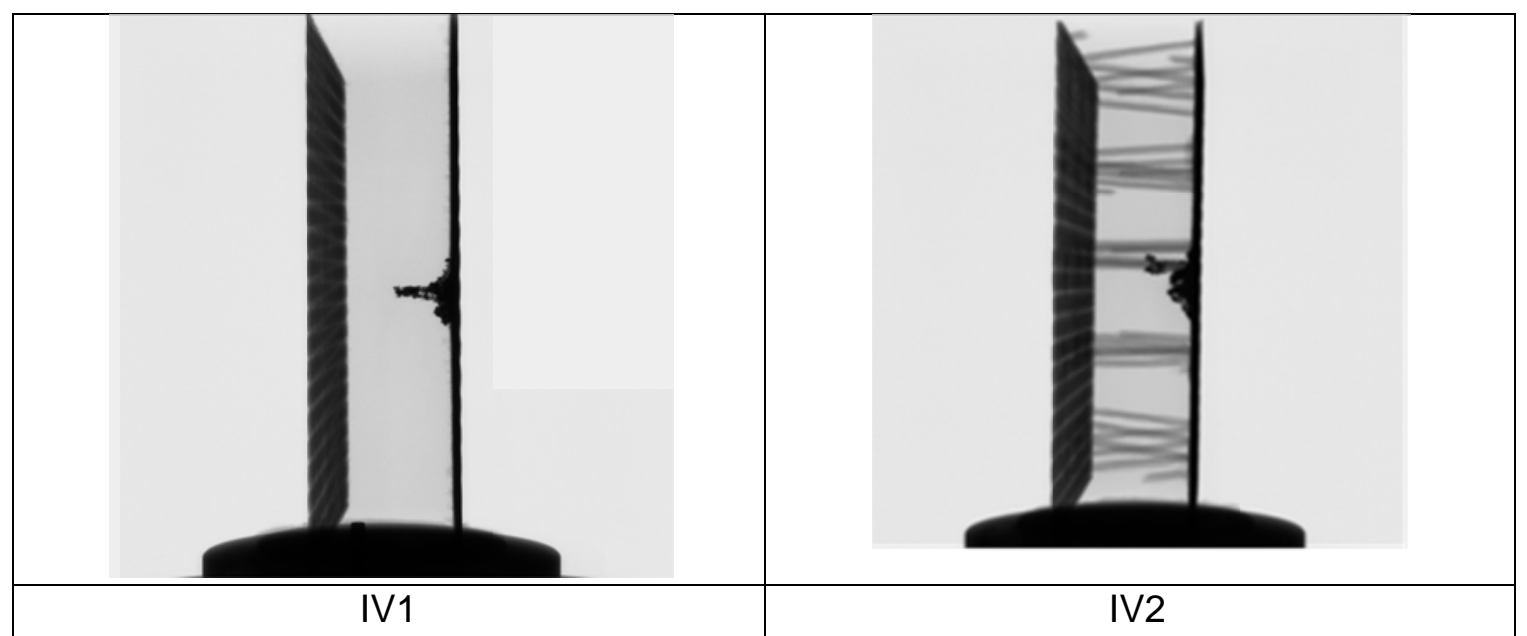

Figure 16.X- ray lateral photographs of the different typologies of glass fiber face sandwich without (left) and with (right) tufting

\section{Conclusions}

Tufting is a simple methodology that allows the insertion of fibers through the thickness of sandwich structures. This methodology has different parameters that can be modified in order to design the structure as a function of the load requirements. Tufted sandwich panels may be fabricated successfully using VARTM processing method with high quality. Nevertheless, this process increases the mass of the sandwich because the resin fill all the tunnels left during the tufting process, therefore a compromise between the performance and the mass of the panel must be found.

Tufted sandwich structures were tested under low-velocity impact. The carbon fiber sandwiches showed that the absorbed energy increased with the 
increasing of the tufting. Similarly, for the glass fiber sandwiches, an increase in absorbed energy is reported as the tufting density increase.

\section{References}

1. A. Henao, M. Carrera, A. Miravete, L. Castejon, Mechanical performance of through-thickness tufted sandwich structures, Composite Structures, Volume 92, Issue 9, August 2010, Pages 2052-2059.

2. E. M. Reis, S. H. Rizkalla. Material characteristics of 3-D FRP sandwich panels. Construction and Building Materials 22 (2008) 1009-1018.

3. S. C. Sharma et al. Buckling Response of Stitched Polyurethane Foam Composite Sandwich Structures. Journal of Reinforced Plastics and Composites 2004; 23; 1267.

4. B. Lascoup et al. On the mechanical effect of stitch addition in sandwich panel. Composites Science and Technology 66 (2006) 1385-1398.

5. L. Tong et al. 3D Fiber Reinforced Polymer Composites. First edition, elsevier science Ltd. UK, 2002.

6. M.C. de Verdiere. Effect of tufting on the response of non crimp fabric composites. ECCOMAS Thematic Conference on Mechanical Response of Composites. Porto, Portugal, 12-14 september 2007.

7. A.P. Mouritz et al. A review of the effect of stitching on the in-plane mechanical properties of fiber-reinforced polymer composites. Conqwsires Part A 28A (1997) 979-99

8. C. Scarponi et al. Advanced TTT composite materials for aeronautical purposes: Compression after impact (CAI) behavior. Composites: Part B 38 (2007) 258-264.

9. C. Sickinger, A. Herrmann. Structural Stitching as a Method to design High- Performance Composites in Future. Institute of Structural Mechanics. GermanAerospaceCenter. Germany

10.T. Thurm. Application areas of one-side stitching technique. ALTIN Nähtechnik GmbH. Germany.

11.P. Potluri et al. Novel stitch-bonded sandwich composite structures. Composite Structures 59 (2003) 251-259. 